\title{
An Extended CMOS ISFET Model Incorporating the Physical Design Geometry and the Effects on Performance and Offset Variation
}

\author{
Yan Liu, Student Member, IEEE, Pantelis Georgiou, Member, IEEE, Themistoklis Prodromakis, Member, IEEE, \\ Timothy G. Constandinou, Senior Member, and Christofer Toumazou, Fellow, IEEE
}

\begin{abstract}
This paper presents an extended model for the CMOS-based Ion-Sensitive-Field-Effect-Transistor (ISFET), incorporating design parameters associated with the physical geometry of the device. This can, for the first time, provide a good match between calculated and measured characteristics by taking into account the effects of non-idealities such as threshold voltage variation and sensor noise. The model is evaluated through a number of devices with varying design parameters (chemical sensing area and MOSFET dimensions) fabricated in a commercially-available $0.35 \mu \mathrm{m}$ CMOS technology. Threshold voltage, subthreshold slope, chemical sensitivity, drift and noise were measured and compared to the simulated results. The first and second order effects are analysed in detail and it is shown that the sensors' performance was in agreement with the proposed model.
\end{abstract}

Index Terms-ISFET, CMOS, geometry, drift, noise, chemical sensor, threshold voltage, subthreshold slope, passivation capacitance

\section{INTRODUCTION}

$\mathbf{T}$ HE Ion-Sensitive-Field-Effect-Transistor (ISFET) was first introduced by Bergveld in the 1970s [1] and since then has been used widely in numerous sensing applications [2]-[4]. In recent years, the ISFET has been implemented in commercially-available CMOS technologies [3], [5]. Implementation in CMOS is highly desirable due to the advantages of significantly reduced manufacturing complexity and therefore cost, as well as the option for integration, i.e together with instrumentation or in large sensor arrays [4], [6]. However CMOS based ISFETs suffer from a number of non-ideal characteristics [3], [7]-[9], such as threshold voltage variation, drift and noise. In recent years there has been increasing interest in investigating the source of these effects and although the fundamental underlying mechanisms are understood, there has been little effort in characterizing and minimizing these [7], [9], [10]. Furthermore, although ISFETs have been fabricated in CMOS for a variety of physical geometries [5], [7], [11], [12], it has not yet been reported how design dimensions impact sensor characteristics. This is a key challenge in designing chemical sensors with reduced dimensions, which are particularly useful in applications such

Manuscript received XXX, XXX; revised XXX, XXX.

All authors are with the Centre for Bio-Inspired Technology, Department of Electrical and Electronic Engineering and Institute of Biomedical Engineering, Imperial College of Science, Technology and Medicine, London SW7 2AZ, United Kingdom. (e-mail:yan.liu06@imperial.ac.uk) as large-scale, highly-integrated chemical sensor arrays [4], [6].

In this paper we present an extended model for CMOSbased ISFETs to include both the first order effects, (i.e. intrinsic dimension-related characteristics) and second order effects, (i.e. non-linear characteristics). By focusing on the effect of varying the design parameters (i.e physical dimensions) a capacitance-based model is derived which includes all capacitive structures, the values of which are directly related to physical dimensions. Based on this, threshold voltage, subthreshold slope, chemical transconductance, drift and noise are analyzed to establish a extended model for CMOS ISFETs. A test chip, including six specific devices with varying coreMOSFET $(W / L)$ and the chemical sensing area $\left(W_{c} / L_{c}\right)$ has been prototyped to evaluate this model. The measured results demonstrated a good agreement between the proposed model and the performance of fabricated sensors.

The paper is organized as follows: Section II proposes the extended CMOS ISFET model incorporating all the design parameters, while Section III explains the research methodology and the sensors implementation. Section IV presents measured results with detailed discussion on both the first and the second order effects. Finally Section V concludes this study and indicates how this model can aid sensor and readout circuit design with reduced calibration effort.

\section{AN EXTENDED CMOS ISFET MODEL}

\section{A. Overview}

Traditionally, ISFETs devices have been fabricated as MOSFET devices with the gate metal and oxide being replaced by an insulating sensing membrane [1]. CMOS ISFETs are fabricated by extension (i.e. electrical connection) of the MOSFET poly-silicon gate (IPG) to the top metal layer [3], [5], utilizing the intrinsic passivation as the sensing membrane, as illustrated in Fig. 1. Compared to an intrinsic MOSFET device, a CMOS-ISFET is essentially a floating-gate MOSFET with one floating remote gate voltage influenced by the reference voltage and electrochemical potential. Therefore in a similar manner to the MOSFET model, the drain current of the ISFETs can be represented as a function of the floating gate voltage $V_{F G}$ and design dimensions [10]. $V_{F G}$ is modulated by the chemical potential $V_{\text {chem }}$ and the voltage bias applied on the reference electrode. $V_{\text {chem }}$ is a combination of the potential drop between the interface and $\mathrm{pH}$ induced potential, 


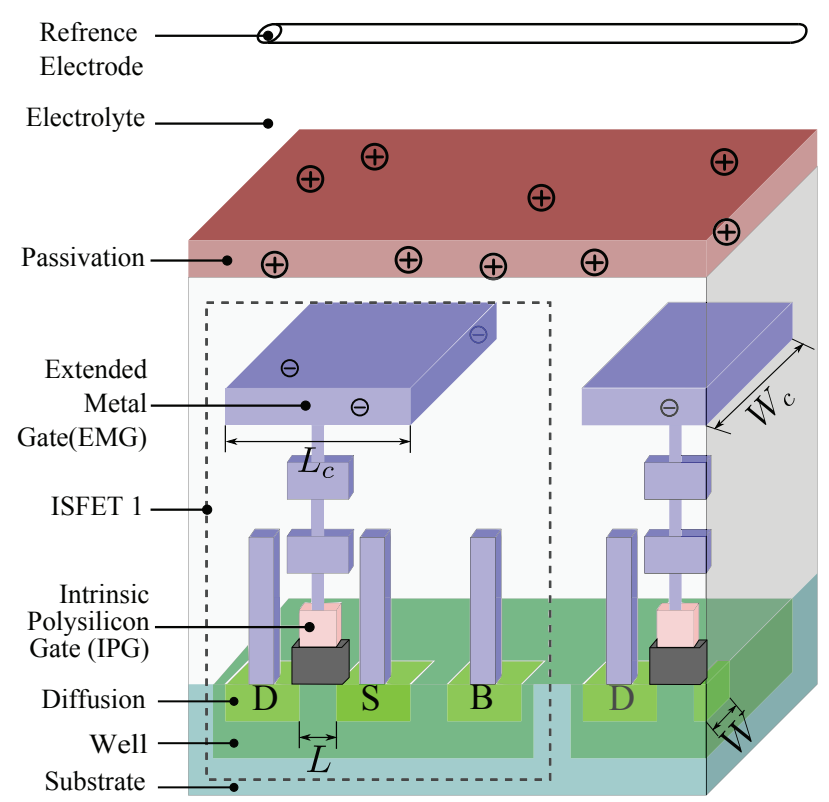

Fig. 1: CMOS ISFET structure.

as given in [3]. Since the potential of the reference electrode must remain constant with varying $\mathrm{pH}$, the potential across the electrolyte-insulator is the only value influenced by change in $\mathrm{pH}[3]$.

The chemical gate voltage, and the electrical terminal voltages are coupled to IPG via different series of capacitors. These capacitors exhibit non-linear effect on the overall sensor performance such as variation of capacitance [12] and trapped charge [13], [14]. Among them, passivation capacitance $\left(C_{\text {pass }}\right)$ is of great importance, it couples the chemical potential and then influence sensor chemical response directly. In [10], a basic CMOS ISFET model based on passivation capacitance for weak inversion was proposed illustrating transconductance efficiency reduction compared to the corresponding MOSFET characteristic. In this work, we develop a more complete model that focuses on the impact of the stacked capacitance on the sensor characteristics and the relationship between capacitance and design dimensions $\left(W / L\right.$ and $\left.W_{c} / L_{c}\right)$.

Fig. 2 illustrates the proposed model that incorporates the various capacitances including trapped charge to analyze the electrical performance of the devices. The parameters are detailed in Appendix A. This model includes both the intrinsic and parasitic capacitors and provides a clear relationship between biasing voltage and floating gate voltage. We intentionally omit the parasitic capacitance seen from either floating gate or passivation to the channel, since this capacitance is relatively small compared to the gate-oxide capacitance and is therefore negligible. The faradaic impedance of the electrolyte and reference electrode is also neglected in this model, since they have negligible effect on the electrical performance of the devices. Parasitic capacitances coupled to the floating gate include two parts: overlap capacitance within the transistor, and extrinsic parasitics caused by extended metal gate(EMG). The first value is embedded within the MOSFET model, while the second term can be extracted by either simulation tools

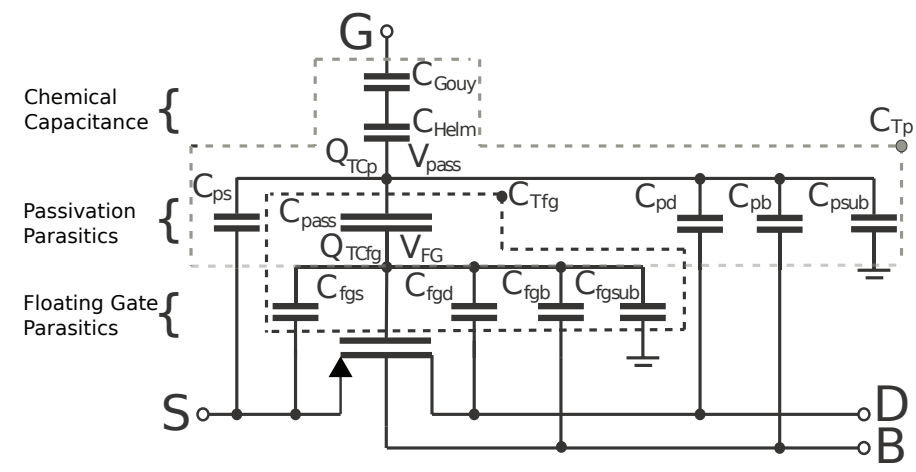

Fig. 2: CMOS ISFET stack capacitors model

or experiments [15]. At the passivation node the parasitic capacitances can be estimated by the simple parallel plate capacitor model, resulting in a relatively smaller than the chemical capacitance $C_{\text {Gouy }}$ and $C_{H e l m}$ [10], [16].

When modelling the passivation capacitance of an ISFET, a simple parallel plate capacitance model is not sufficient, since the corresponding fringing fields are considerably large due to the large metal to dielectric thickness ratio. Detailed analytical derivation is beyond the scope of this paper. Instead, we used a finite element analysis tool (Ansoft Maxwell 3D) [17] to simulate a simple chemical sensing area model, using $\mathrm{SiO}_{2}$ and $\mathrm{Si}_{3} \mathrm{~N}_{4}$ as the dielectric medium and two aluminium plates as the electrodes, representing the floating metal and electrolyte. Depending on the FEA (Finite Element Analysis) results, the passivation capacitance for a common CMOS technology with $\mathrm{SiO}_{2}$ and $\mathrm{Si}_{x} N_{y}$ as passivation layer can be simplified to:

$$
\begin{aligned}
C_{\text {pass }} & =C_{\mathrm{SiO}_{2}}+C_{\mathrm{Si}_{x} N_{y}} \\
& =\frac{\varepsilon_{\mathrm{SiO}_{2}} \varepsilon_{\mathrm{Si}_{x} N_{y}}}{\varepsilon_{\mathrm{SiO}_{2}} d_{\mathrm{Si}_{x} N_{y}}+\varepsilon_{S i_{x} N_{y}} d_{\mathrm{SiO}_{2}}}\left(W_{c} L_{c}\right)^{\beta}
\end{aligned}
$$

Where $\varepsilon_{S i O_{2}}$ and $\varepsilon_{S i_{x} N_{y}}$ are the dielectric constant, $\mathrm{d}$ is the thickness for corresponding layers and $\beta$ is the chemical area scaling factor due to non-linear effect such as the fringing field. Therefore, the drain current of ISFETs can be represented by a function of design dimensions, biasing, ion concentration (i.e. $\mathrm{pH}$ in this paper) and ISFET threshold voltage:

$$
I_{D}=f\left(W, L, W_{C}, L_{C}, V_{D}, V_{S}, V_{B}, V_{r e f}, p H, V_{\text {thISFET }}\right)
$$

In order to incorporate the sensors into a measurement system, the threshold voltage, transconductance, drift and noise are required for readout or calibration circuits design, and will be discussed in the following sections.

\section{B. Threshold Voltage}

The ISFET threshold voltage $V_{\text {thISFET }}$ is defined as the remote gate to the source voltage required for turning on the underlying transistor. This value, however, commonly exhibits a non-pH related large threshold voltage variation [3], [10]. This can prevent its operation when standard supply voltages are used, in addition to limiting output resolution and accuracy. Reasons for this effect are reported to be due to trapped charge either within the passivation layer and/or the EMG connected 
For the passivation:

$C_{p a s s}\left(V_{F G}-V_{p a s s}\right)+C_{c h e m}\left(V_{\text {ref }}+V_{c h e m}-V_{p a s s}\right)+C_{p s}\left(V_{S}-V_{p a s s}\right)+C_{p b}\left(V_{B}-V_{p a s s}\right)+C_{p d}\left(V_{D}-V_{p a s s}\right)+C_{p s u b}\left(V_{s u b}-V_{p a s s}\right)=Q_{T C p}(3)$

$Q_{T C p}$ is the trapped charge within the passivation; $V_{\text {pass }}$ is the potential drop across the passivation layer; $V_{r e f}$ is the reference voltage in bulk solution and $V_{s u b}$ is the substrate voltage.

For the floating gate:

$$
C_{p a s s}\left(V_{p a s s}-V_{F G}\right)+C_{f g s}\left(V_{S}-V_{F G}\right)+C_{f g d}\left(V_{D}-V_{F G}\right)+C_{f g b}\left(V_{B}-V_{F G}\right)+C_{f g s u b}\left(V_{s u b}-V_{F G}\right)+C_{o x}\left(\psi_{s a}-V_{F G}\right)=Q_{T C f g}
$$

$Q_{T C f g}$ is the trapped charge within the floating gate; $\psi_{s a}$ is the surface potential of the channel.

$$
\begin{aligned}
& V_{F G}=\left[\left(C_{\text {chem }}\left(V_{\text {ref }}+V_{\text {chem }}\right)+\sum C_{i} V_{i}+Q_{T C p}\right) \frac{C_{p a s s}}{C_{T p}}+\sum C_{j} V_{j}+Q_{T C f g}\right] \frac{C_{T p}}{C_{T p} C_{T f g}-C_{p a s s}^{2}} \\
& V_{\text {th-ISFET }}=\underbrace{\frac{V_{t h-M O S F E T} C_{T f g} C_{T C p}}{C_{\text {chem }} C_{\text {pass }}}}_{\text {MOSV } V_{\text {th }} \text { contribution }}+\underbrace{\frac{\left(V s C_{T f g}-\sum C_{j} V_{j}\right) \frac{C_{T C p}}{C_{p a s s}}-\sum C_{i} V_{i}}{C_{\text {chem }}}}_{\text {Parastic contribution }}-\underbrace{\frac{Q_{T C f g} \frac{C_{T C p}}{C_{p a s s}}+Q_{T C p}}{C_{c h e m}}+\underbrace{\kappa}_{\text {Chemical contribution }}}_{\text {Trapped charge contribution }}
\end{aligned}
$$

to the IPG [11]. UV irradiation [18] and hot electron injection [11] have been used to remove this and thus reduce any threshold mismatch.

In order to determine the origin of the threshold voltage variation, charge equilibrium is established on the floating nodes (both the floating gate and passivation), as described in equations (3) and (4), then the floating gate voltage of the device was derived, shown in Eq. (5). The floating gate voltage $V_{F G}$ and threshold voltage for the MOSFET $V_{t h}$, as described in [19], can be combined to form Eq. (6). It should be noted that the $\mathrm{pH}$-related term is accounted for in $\kappa$, which contains $V_{\text {chem }}$ and $C_{\text {chem }}$ [20], [21]. In this section only the first order DC characteristics are considered and therefore it can be assumed that $\kappa$ and $C_{c h e m}$ are constant.

From Eq. (6), it can be seen that the four factors influencing the threshold voltage of the device are: the intrinsic $V_{t h}$ of the MOSFET enhanced by the capacitance ratio, the parasitic effect of constant biasing due to floating gate nodes, the trapped charge within both the floating gate stack and the passivation; the chemical related constant $\kappa$. Simulated results using the proposed model with a sensor dimension of $10 \mu \mathrm{m}$ $\times 10 \mu \mathrm{m}$ are shown in Fig. 3. It can be found that for large chemical area and terminal voltages, the $V_{\text {thISFET }}$ increases non-linearly.

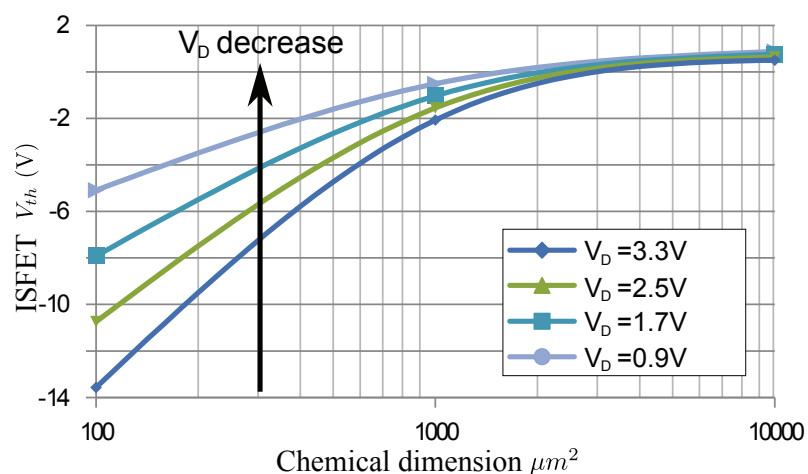

Fig. 3: Threshold voltage simulation results. The solid lines show $V_{t h}$ change with regards to $V_{D}$ and chemical dimensions, when $V_{S}=3.3 \mathrm{~V}$ for a $10 \mu \mathrm{m} \times 10 \mu \mathrm{m}$ electrical area.

\section{Transconductance and Subthreshold slope}

Due to the existence of passivation capacitance, the transconductance of ISFETs seen referring to the remote gate are scaled down compared to MOSFETs with identical design dimensions. By combining Eq. (5) and transconductance in saturation for MOSFET [19], the transconductance of ISFET can be derived as:

$$
g m_{I S F E T}=k^{\prime} \frac{C_{\text {chem }} C_{\text {pass }}}{C_{T p} C_{T f g}-C_{\text {pass }}^{2}} \frac{W}{L}
$$

By assuming that the depletion capacitance remains constant in deep weak inversion, the subthreshold slope can be represented by:

$$
\begin{aligned}
\text { Sub_slope } & =\frac{d V_{G S}}{d\left(\log I_{D S}\right)}=\frac{d V_{G S}}{d V_{\text {pass }}} \frac{d V_{\text {pass }}}{d V_{F G}} \frac{d V_{F G}}{d\left(\log I_{D S}\right)} \\
& =2.3 n U_{t}\left(\frac{C_{T p}}{C_{\text {chem }}}+\frac{C_{\text {pass }}}{C_{\text {chem }}} \frac{C_{\text {pass }}}{C_{T f g}}\right) \frac{C_{T f g}}{C_{\text {pass }}}
\end{aligned}
$$

\section{Chemical Transconductance}

In addition to electrical transconductance reduction, the chemical transconductance is scaled compared to custom ISFET sensors [22], which is given by:

$$
g_{p H}=2.3 \alpha V_{t} k^{\prime} \frac{C_{\text {chem }} C_{\text {pass }}}{C_{T p} C_{T f g}-C_{\text {pass }}^{2}} \frac{W}{L}
$$

where $\alpha$ is the scaling factor due to double layer model [16]. This value indicates a non-linear reduction effect between the chemical transconductance of CMOS ISFETs and custom made ISFETs .

\section{E. Drift and Noise}

Due to the poor quality of the passivation layer as sensing membrane, CMOS-based ISFETs suffer from many non-ideal effects, including: drift, noise and temperature instability. The drift mechanism has been described as the dispersive transportation [9], also combined with other effects, such as leakage across the reference electrode [8]. In general, the variation of the surface hydrated layer thickness changes the effective 
thickness of the passivation, hereby altering the potential drop across the insulator:

$$
\Delta V_{G}(t)=-\left(\frac{\varepsilon_{i n s}-\varepsilon_{S L}}{\varepsilon_{i n s} \varepsilon_{S L}}\right) \chi_{S L}(t) \sum Q
$$

where $\sum Q$ is both intrinsic and trapped charge in this structure, $\varepsilon_{\text {ins }}$ and $\varepsilon_{S L}$ are the dielectric constants of $\mathrm{pH}$ sensing membrane and modified layer respectively, while $\chi_{S L}$ represents the depth change of this layer. This change is essentially applied at the remote gate, which can be combined with Eq.(7), causing long term sensors output drift.

Although ISFET thermal noise is present in a wide spectrum, within the chemical signal spectrum, the flicker-like noise dominates and alias slow chemical response. Low frequency noise is studied in [7], [8], [23], in which a 1/f noise pattern was found when the gate leakage is lower than $1 \mathrm{nA}$. It was stated that the measured noise indicates the intrinsic MOSFET noise, provided the gate leakage through the reference electrode was minimal [8]. Moreover, it is believed that the ISFET has similar noise power compared to the MOSFET flicker noise [8].

In similar fashion to CMOS MOSFETs, we propose that ISFETs flicker-like noise is due to both intrinsic channel flicker noise and chemical noise:

$$
\begin{aligned}
\overline{V_{n}^{2}(\text { ISFET })} & =\overline{V_{n}^{2}(M O S F E T)}+\overline{V_{n}^{2}(\text { Chem })} \\
& =\left(\frac{C_{T f g}}{C_{\text {pass }}}\right)^{2} \frac{K_{f}}{W L C_{o x}^{\prime} f}+\frac{K}{f}
\end{aligned}
$$

Where $K$ is the chemical related effect due to long-term electrode degradation and surface chemical noise. A qualitative study is provided in section IV.

\section{Methodology}

In order to evaluate the proposed model, a series of CMOS ISFETs with varying transistor channel size and chemical sensing area need to be fabricated and measured. Since the passivation capacitance is the essential parameter, Eq. (1) can be evaluated by $C_{\text {pass }}$ calculated by the subthreshold slope using Eq. (8), whilst the parasitic and gate insulator capacitance are either simulated or measured from fabricated devices. The threshold voltage of individual sensors can then be derived using the proposed model and compared against the measured results. Evaluation of drift and noise characteristics can be performed by long-term measurement. Furthermore MOSFETs of comparable same electrical dimension need to be fabricated on-site to provide an authoritative comparison with the traditional MOSFET model. The two following subsections detail the methodology with respect to the sensor development and experimental setup.

\section{A. Sensor Implementation}

All ISFET devices presented in this paper have been fabricated in a commercially available $0.35 \mu \mathrm{m} 2 \mathrm{P} 3 \mathrm{M}$ CMOS technology. P-type MOSFETs have served as the core devices for all ISFETs for establishing a $V_{S B}=0$ to minimize any body-effects. A continuous metal stack has been used to couple the IPG to the top metal layer [5]. The passivation layer of this technology, consisting of $\mathrm{SiO}_{2}$ and $\mathrm{Si}_{x} \mathrm{~N}_{y}$, was used to sense $p H$ Eq.(2) with total thickness of $22 \mu \mathrm{m}$. For characterization purposes, all the bulk and source terminals were tied together in a common source terminal. This scheme has been employed since the drain current is the only quantity requiring investigation.

Two sets of electrical dimensions and three sets of chemical dimensions have been used, resulting in a total of six different combinations. To distinguish between the various sensors, the devices were labelled as shown in Table I. Fig. 4 is a the microphotograph of the fabricated sensors with Fig. 4.b showing the cross section. The pitch between adjacent sensors has been set to twice the chemical dimension to minimize any crosstalk. MOSFETs of identical dimensions have also been fabricated on the same die.

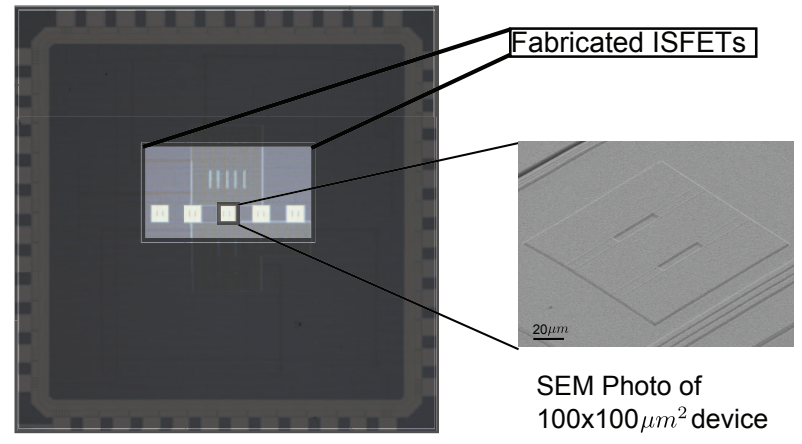

Fig. 4: Microphotograph of Chip layout

TABLE I: ISFETs dimension and name coding

\begin{tabular}{|c|c|c|c|c|}
\hline Electrical W $\times \mathrm{L}$ & \multicolumn{3}{|c|}{ ISFET devices short names } & MOS \\
\hline $100 \times 1 \mu \mathrm{m}^{2}$ & $\mathrm{D} 1 \_1$ & $\mathrm{D} 1 \_2$ & D1_3 & PMOS_1 \\
\hline $10 \times 10 \mu \mathrm{m}^{2}$ & D2_1 & D2_2 & D2_3 & PMOS_2 \\
\hline Chemical $W_{c} \times L_{c} \mu \mathrm{m}^{2}$ & $10 \times 10$ & $100 \times 10$ & $100 \times 100$ & N/A \\
\hline
\end{tabular}

\section{Experimental setup}

After fabrication, the chips were directly mounted onto a printed circuit board (PCB), wire-bonded and encapsulated [24] with the sensing areas clearly exposed. Both the drain and common source of the sensors were connected to a semiconductor characterization system (SCS) Keithley 4200, and ESD protection was provided by using a source measure unit (SMU) Keithley 2602. During the test, the packaged sensors were immersed into electrolytes of known $\mathrm{pH}$ values. $\mathrm{An} \mathrm{Ag} / \mathrm{AgCl}$ reference electrode was used as the remote gate, providing the gate reference voltage through the electrolyte. A calibrated $\mathrm{pH}$ meter was also immersed into the electrolyte alongside the DUT (Device Under Test) to monitor the $\mathrm{pH}$ change for comparison against the ISFETs results. A magnetic stirrer was used to ensure a uniform distribution of ions in the liquid. The entire experimental setup was enclosed within a Faraday cage, to shield environmental electrical noise. The temperature of the electrolyte during the experiments was maintained at $\mathrm{T}=27^{\circ} \mathrm{C}$.

\section{RESUlTS AND VALIDATION OF THE MODEL}

\section{A. Overview}

The overall electrical functionality of the ISFETs is proved by $I_{D}-V_{G S}$ sweep, which are shown in Fig. $5 \mathrm{a}$ and 5 b, 
with the corresponding MOSFET devices as references. The

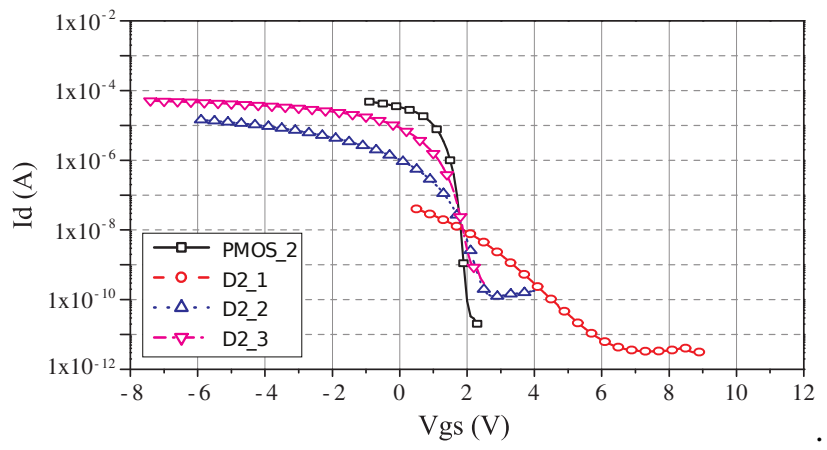

(a)

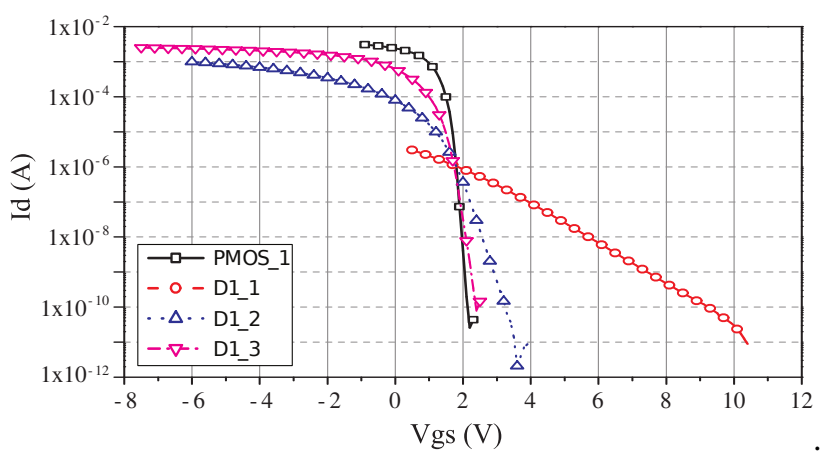

(b)

Fig. 5: $I_{D} / V_{G S}$ curves for CMOS ISFETs of different chemical sensing area with underlying electrical a. W/L $=100 / 1 \mu \mathrm{m}$ and b. $\mathrm{W} / \mathrm{L}=10 / 10 \mu \mathrm{m}$

relevant chemical responses are shown in Fig. 6, in which the ISFET output current tracks the $\mathrm{pH}$ change continuously. The overall chemical responses referred to the gate voltage are also listed in Table II.

TABLE II: ISFETs $\mathrm{pH}$ sensitivity

\begin{tabular}{|c|c|c|c|c|}
\hline $\begin{array}{l}\text { Electrical } \\
(\mathrm{W} \times \mathrm{L})\end{array}$ & $\begin{array}{l}\text { Average } \\
(\mathrm{mV} / \mathrm{pH})\end{array}$ & chemical & sensitivity & Total \\
\hline $100 \times 1 \mu m^{2}$ & 33.58 & 52.77 & 33.33 & $\mu=35.5^{\circ}$ \\
\hline $10 \times 10 \mu m^{2}$ & 26.95 & 34.12 & 42.59 & $\sigma=14.04$ \\
\hline$W_{c} \times L_{c} \mu m^{2}$ & $10 \times 10$ & $100 \times 10$ & $100 \times 100$ & \\
\hline
\end{tabular}

\section{B. First order effects}

1) Subthreshold slope: The subthreshold slope can be extracted from the IV sweep, which are listed in Table. III. What can be observed is that the subthreshold slopes of the ISFET devices are much larger than those of the corresponding MOSFETs. Additionally these values are observed to be inversely proportional to the chemical sensing areas.

TABLE III: Sub-threshold slopes of ISFETs and corresponding MOSFETs

\begin{tabular}{|c|c|c|c|c|}
\hline Electrical W $\times$ L & ISFET devices subthreshold slope & MOSFET \\
\hline $100 \times 1 \mu m^{2}$ & 2.003 & 0.343 & 0.160 & 0.079 \\
\hline $10 \times 10 \mu m^{2}$ & 1.248 & 0.289 & 0.400 & 0.079 \\
\hline$W_{c} \times L_{c} \mu m^{2}$ & $10 \times 10$ & $100 \times 10$ & $100 \times 100$ & N/A \\
\hline
\end{tabular}

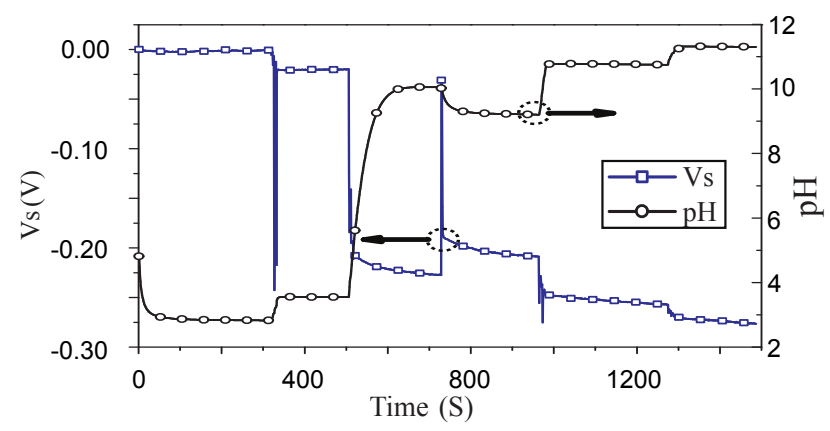

(a)

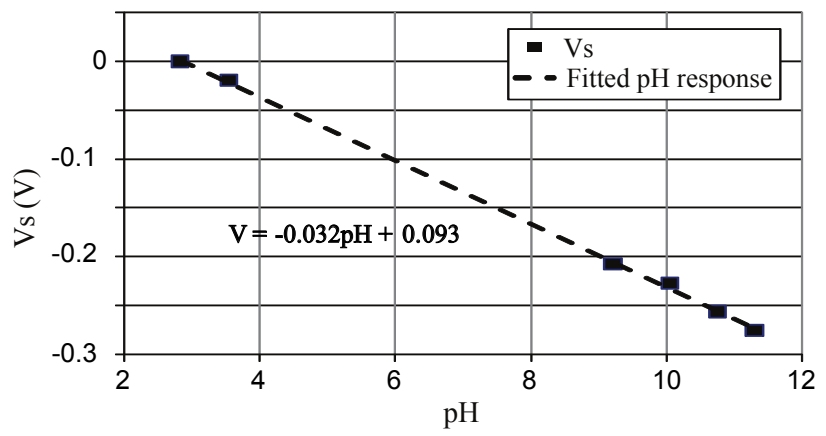

(b)

Fig. 6: ISFET pH response. Shown are: (a) ISFET output versus $\mathrm{pH}$ in time domain and (b) ISFETs $\mathrm{pH}$ sensitivity extracted from linear fitting.

2) Passivation capacitance: By using Eq. (8), the passivation capacitance, based on measured results, is calculated and illustrated in Fig. 7 for an increasing chemical area. Simulated results using Eq. (1) and FEA tool are shown in the same graph as a reference. The geometric parameters used in FEA tool are identical as the physical dimension of the sensors.

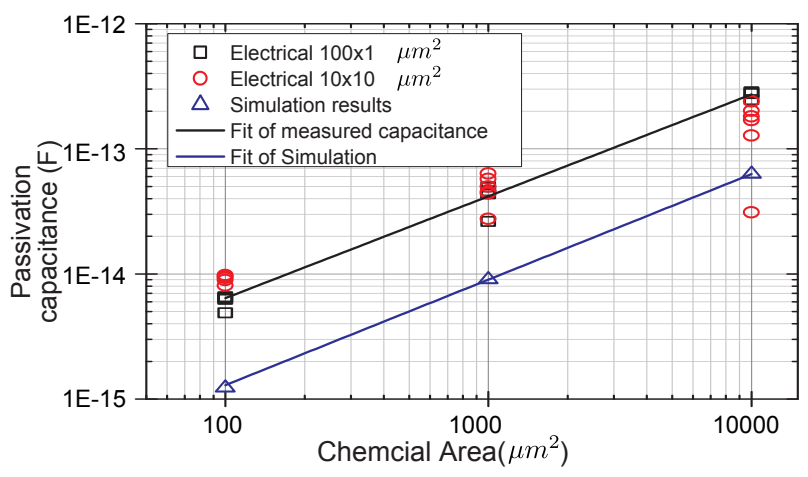

Fig. 7: Extracted passivation capacitance

Using a polynomial fit to the experimental data, this indicates that the effective capacitance increases by chemical area by a power of 0.7. This is in agreement with FEA simulated results. The only discrepancy was a shift in absolute capacitance. Possible reasons include inaccurate estimation of the dielectric medium thickness (due to the surface abrasion during chemical mechanical polishing) and/or parasitics omitted from the previous assumption. Moreover, the included parasitic capacitance coupled to the floating gate can be much bigger than the EDA simulated results. 
3) Threshold voltage: Using the constant current method described in [25], the threshold voltages of the devices were extracted. The same method was also used for the intrinsic MOSFET devices, whose $V_{t h}$ was consistent with the simulation results based on the BSIM $3 \mathrm{v} 3$ model [26]. The current limit to detect threshold voltage is $1 \mu \mathrm{A}$ for $100 \mu \mathrm{m} \times 1 \mu \mathrm{m}$ electrical dimensions and $100 \mathrm{nA}$ for $10 \mu \mathrm{m} \times 10 \mu \mathrm{m}$ devices.

Fig. 8 exhibits the distribution of threshold voltage of all the fabricated sensors when exposed to a $\mathrm{pH} 7$ buffer. The threshold voltages were found to be distributed across a broad range of $-14 \mathrm{~V}$ to $+8 \mathrm{~V}$, without any obvious statistical correlation. This is believed due to trapped charge and parasitic effect shown in Eq.(6). For comparison, the corresponding MOSFET devices exhibited consistent threshold voltage of around $0.6 \mathrm{~V}$ with small deviation (standard expected mismatch). Fig. 9 compares measured results (solid dots) with

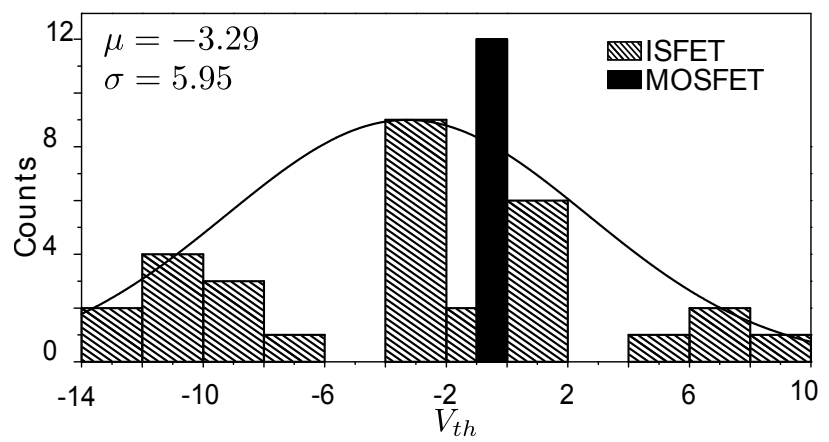

Fig. 8: Variation of threshold voltage of characterized CMOS ISFETs compared to their MOSFET counterparts.

calculated threshold voltage (dashed area) using the proposed model, in which a fairly good compliance can be found. The dashed area also illustrates the trapped charge error, which will be discussed under the second order effects section.

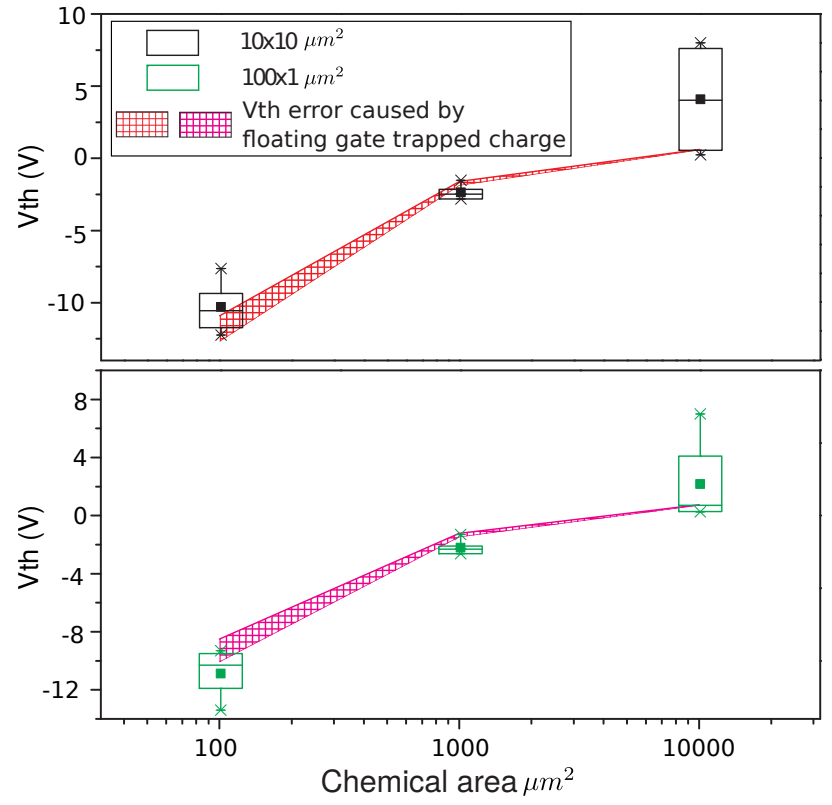

Fig. 9: Threshold voltage range versus chemical area

4) Drift and noise: It was found that the drift of CMOSbased ISFETs had a negligible dimensional relationship to the physical dimensions. The drift distribution and average drift are illustrated in Fig. 10, with the red line indicating the corresponding MOSFETs. It is evident that the drift of ISFETs is distributed from $1.5 \mathrm{mV}$ to $8.5 \mathrm{mV}$ per hour (with average results of $5.8 \mathrm{mV} / \mathrm{hr}$ ). This is at least 3 times larger than the corresponding MOSFETs (which had $0.2 \mathrm{mV} / 2000 \mathrm{sec}$ ). The trend of ISFETs drift exhibited a relaxed-exponential characteristic, which is in accordance with Eq. (10).

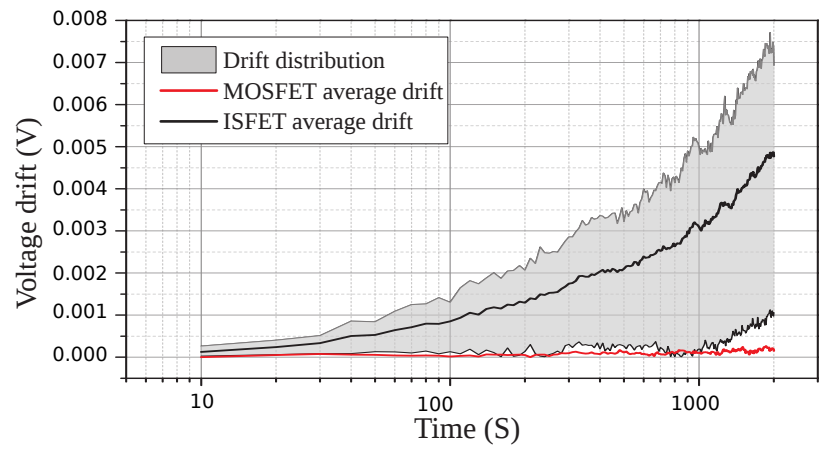

Fig. 10: Long term drift of ISFETs and MOSFETs fabricated on the same process.

Fig. 11 illustrates the low frequency noise, where the dashed line indicates the simulation results of corresponding MOSFETs, and the solid line illustrates the measured results of MOSFETs fabricated on the same die. The scattered dots show the measured low frequency noise, with red dots indicating the average value and grey area for the noise distribution. It was found that the measured MOSFET noise is 1 order of magnitude larger than simulation results. This can be attributed to the localized temperature drift and measurement system noise. This noise level is considered to be the baseline of this measurement system. There can be seen no clear relationship between noise magnitude and chemical sensing area within the devices tested. Therefore, according to the Eq. (11), the chemical flicker-like noise dominates within the tested spectrum. From empirical observation throughout our specimens, we estimate $\mathrm{K}=1 \mathrm{n} V^{2} \mathrm{~Hz}$ severing as possible detection limit of ISFETs measurement limit, as well as the average exhibited noise floor. This confines that applications requiring measurement accuracy of $5 \mathrm{mV} / \mathrm{hr}$ or lower can indeed be very challenging.

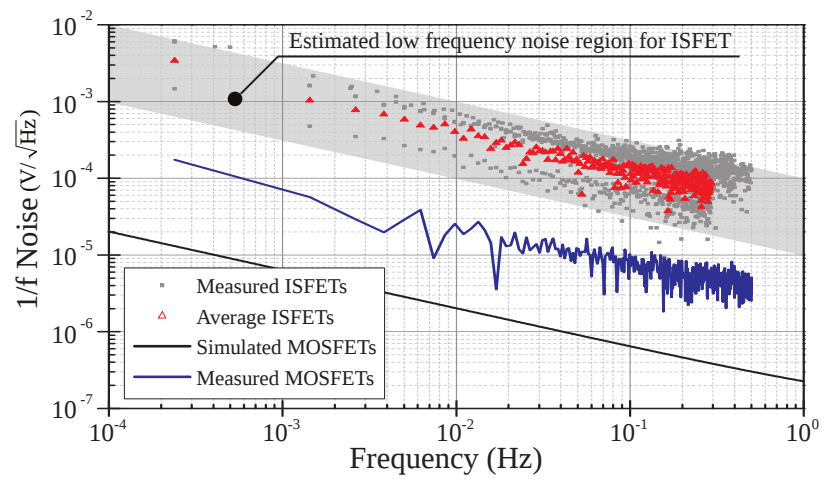

Fig. 11: Low frequency noise of ISFETs with identical electrical area. 


\section{Second order effects}

This paper has largely focused on the first order effects, while non-ideal factors are also considered and included in the proposed model. This final section discussed second order effect with comparison to the experiment's results.

1) Chemical capacitance: The chemical capacitance, including double layer capacitance and Faradic impedance is not directly related to the design parameters. However, in low frequency spectrum, where most chemical reaction taking place, the Warburg capacitance effect in the proposed model is negligible [27]. According to [28], the chemical capacitance $C_{c h e m}$ including $\mathrm{Helm}$ capacitance $C_{\mathrm{Helm}}$ (the double plate capacitance) and Gouy capacitance $C_{\text {Gouy }}$, (the double layer distribution capacitance) is approximately $1 \mathrm{pF} / \mu \mathrm{m}^{2}$. Considering $C_{p s}+C_{p d}+C_{p s u b}$ as a parallel plate capacitor, from top passivation to the substrate, the chemical capacitance is at least 3 orders of magnitude larger than the sum of all other parasitic capacitances. Therefore, it has negligible effect in Eq. (7), (8), and (6).

2) Trapped charge: There are two possible mechanisms of trapping: floating gate charge, and dielectric trapped charge on the passivation. In the floating gate it is caused by residual electrons during fabrication [29], giving an offset $V_{t h F G M O S}$ of a several hundred mili-volts [30]. In the passivation $Q_{T C p}$ is due to intrinsic dangling bonds within $S i_{x} N_{y}$, buried site or surface defects caused by extrinsic dangling bonds [31], [18]. From Eq. (6), and [30], the estimated ISFET $V_{t h}$ variation caused by floating gate trapped charge is illustrated by the shadowed area in Fig. 9. This demonstrates that devices with a small chemical area exhibit offsets of up to 2 Volts. This can be reduced to a negligible level simply by increasing the chemical area.

However, compared to the deviation in the measurement, for larger chemical areas, $Q_{T C p}$ is the more dominant, as the measurement deviation becomes 10 times larger than the estimation. This demonstrates that the effect of trapped charge within the passivation layer can be equivalent or even higher than the effect of trapped charge in the EMG.

\section{CONClusion}

This paper has, for the first time, presented a model for CMOS-based ISFET sensors that incorporates physical design geometry. It has been shown that this model can be used to provide a measure for non-ideal effects such as threshold voltage variation, drift and noise. This model has been verified by testing a number of CMOS-based ISFETs with varying the electrical $(W / L)$ and chemical sensing area $\left(W_{c} / L_{c}\right)$. Measured results show that the sensors do exhibit an inversion region and chemical sensitivity as expected, however, with lower transconductance and sub-threshold slopes when compared to their MOSFET counterparts. The passivation capacitance (extracted from the sub-threshold slope) is in a good agreement with simulation results. Threshold voltage extracted from measured results were in accordance with simulated results with reasonable error due to trapped charge. The measured drift varied from $1 \mu \mathrm{V} / \mathrm{sec}$ to $4 \mu \mathrm{V} / \mathrm{sec}$ and was at least 3 orders of magnitude larger than those for the corresponding
MOSFETs. The low-frequency noise magnitude in the devices has been found to be one order of magnitude larger than those of the intrinsic MOSFETs. Using the model, an empiricallyderived value for the chemical noise is estimated, providing the minimum noise level for fabricated CMOS ISFETs.

Second order effects such as chemical capacitance and trapped charge influence have also been discussed. By comparing the calculated $V_{\text {thISFET }}$ offset, it has been determined that for devices with smaller chemical dimension, the floating gate charge dominates the offset, where in larger devices, the surface dielectric trapped charge is more dominant. By using this model, a design compromise can be made to dramatically reduce sensors offsets and errors. Moreover, a good estimation of threshold voltage, transconductance, and noise can be derived, which eases the design specification for onchip calibration circuits, but also determines the measurement limit for specific ISFET sensors.

\section{APPENDIX}

TABLE IV: Table of Parameters

\begin{tabular}{|l|l|}
\hline \multicolumn{2}{|l|}{ Design and biasing parameters } \\
\hline$W / L$ & MOSFET channel width and length \\
\hline$W_{c} / L_{c}$ & Chemical area dimension(top metal width and length) \\
\hline$V_{F G}$ & Floating gate voltage of metal stacks \\
\hline$V_{S}, V_{D}, V_{B}$ & Source, Drain, Bulk voltage of transistor \\
\hline$V_{s u b}$ & Substrate voltage of the chip \\
\hline Chemical parameters \\
\hline$C_{H e l m}$ & Helm capacitance of intrinsic MOSFET \\
\hline$C_{G o u y}$ & Gouy distribution capacitance of intrinsic MOSFET \\
\hline$C_{c h e m}$ & $C_{H e l m}+C_{G o u y}$ \\
\hline$\kappa$ & Chemical constant with constant pH \\
\hline First order effects related parameters \\
\hline$\psi_{s a}$ & Surface potential of the channel \\
\hline$V_{t h I S F E T}$ & ISFET threshold voltage \\
\hline$I_{D}$ & ISFET drain current \\
\hline$C_{d}$ & Depletion capacitance of MOSFET \\
\hline$C_{o x}$ & Gate dioxide capacitance of MOSFET \\
\hline$C_{p a s s}$ & Passivation capacitance of sensing membrane \\
\hline$C_{p s}$ & Passivation to source parasitic capacitance \\
\hline$C_{p d}$ & Passivation to drain parasitic capacitance \\
\hline$C_{p b}$ & Passivation to bulk/well parasitic capacitance \\
\hline$C_{p s u b}$ & Passivation to substrate parasitic capacitance \\
\hline$C_{T p}$ & Total capacitance associated to the passivation \\
\hline$C_{f g s}$ & Floating gate to source parasitic capacitance \\
\hline$C_{f g d}$ & Floating gate to drain parasitic capacitance \\
\hline$C_{f g b}$ & Floating gate to bulk/well parasitic capacitance \\
\hline$C_{f g s u b}$ & Floating gate to substrate parasitic capacitance \\
\hline$C_{T f g}$ & Total capacitance associated to the floating gate node \\
\hline$C_{i}$ & One of $C_{p s}, C_{p d}, C_{p b}$, or $C_{p s u b}$ \\
\hline$V_{i}$ & Terminal voltages corresponding to individual $C_{i}$ \\
\hline$C_{j}$ & One of $C_{f g s}, C_{f g d}, C_{f g b}$, or $C_{f g s u b}$ \\
\hline$V_{j}$ & Terminal voltages corresponding to individual $C_{j}$ \\
\hline Second order related parameters \\
\hline$Q_{T C p}$ & Trapped charge in passivation \\
\hline$Q_{T C f g}$ & Trapped charge in floating gate \\
\hline
\end{tabular}

\section{REFERENCES}

[1] P. Bergveld, "Development of an ion-sensitive solid-state device for neurophysiological measurements." IEEE Tran. Biomed. Eng., vol. 17, no. 1 , pp. 70-1, 1970.

[2] P. Bergveld and A. Sibbald, Analytical and biomedical applications of ion-selective field-effect transistors. Elsevier, 1988.

[3] P. Bergveld, "Thirty years of ISFETOLOGY What happened in the past 30 years and what may happen in the next 30 years," Elsevier Sensors \& Actuators: B. Chemical, vol. 88, no. 1, pp. 1-20, 2003.

[4] D. Garner et al., "A multichannel DNA SoC for rapid point-of-care gene detection," in Proc. IEEE ISSCC, 2010, pp. 492-493. 
[5] J. Bausells et al., "Ion-sensitive field-effect transistors fabricated in a commercial CMOS technology," Elsevier Sensors \& Actuators: B. Chemical, vol. 57, no. 1-3, pp. 56-62, 1999.

[6] T. Constandinou et al., "A CMOS-based lab-on-chip array for the combined magnetic stimulation and opto-chemical sensing of neural tissue," in Proc. IEEE CNNA, 2010, pp. 1-6.

[7] B. Palan et al., "CMOS ISFET-based structures for biomedical applications," Proc. IEEE Conf. Microtech. Med. Biol., pp. 502-506, 2000.

[8] C. Jakobson et al., "Low frequency noise and drift in ion sensitive field effect transistors," Elsevier Sensors and Actuators B: Chemical, vol. 68, no. 1-3, pp. 134-139, 2000

[9] S. Jamasb et al., "A physical model for drift in pH ISFETs," Elsevier Sensors \& Actuators: B. Chemical, vol. 49, no. 1-2, pp. 146-155, 1998

[10] P. Georgiou and C. Toumazou, "ISFET characteristics in CMOS and their application to weak inversion operation," Elsevier Sensors and Actuators B: Chemical, vol. 143, no. 1, pp. 211-217, 2009.

[11] P. Georgiou and C. Toumazou, "ISFET threshold voltage programming in CMOS using hot-electron injection," IET Electronics Letters, vol. 45, p. 1112, 2009.

[12] T. Prodromaks, Y. Liu, T. Constandinou, P. Georgiou, and C. Toumazou, "Exploiting CMOS Technology to Enhance the Performance of ISFET Sensors," IEEE Electron Device Lett., vol. 31, no. 9, pp. 1053-1055, 2010.

[13] T. Prodromakis et al., "Effect of mobile ionic-charge on CMOS based ion-sensitive field-effect transistors (ISFETS)," Proc. IEEE ISCAS, pp. 2165-2168, 2009

[14] M. Milgrew and D. Cumming, "Matching the Transconductance Characteristics of CMOS ISFET Arrays by Removing Trapped Charge," IEEE Trans. Electron Devices, vol. 55, no. 4, pp. 1074-1079, 2008.

[15] M. Wong et al., "Analysis of the subthreshold slope and the linear transconductance techniques for the extraction of the capacitance coupling coefficients of floating-gate devices," IEEE Electron Device Lett., vol. 13 , no. 11, 1992.

[16] R. van Hal et al., "A novel description of ISFET sensitivity with the buffer capacity and double-layer capacitance as key parameters," Elsevier Sensors \& Actuators: B. Chemical, vol. 24, no. 1-3, pp. 201205, 1995.

[17] Ansoft Maxwell 3D, http://www.ansoft.com/products/em/maxwell/.

[18] P. Hammond et al., "Design of a single-chip $\mathrm{pH}$ sensor using a conventional 0.6-/spl $\mu \mathrm{m}$ CMOS process," IEEE Sensors Journal, vol. 4 , no. 6, pp. 706-712, 2004

[19] Y. Tsividis, Operation and Modeling of the MOS Transistor. McGrawHill, 1987.

[20] P. Bergveld and A. Sibbald, "Comprehensive analytical chemistry, Vol. XXIII," 1988.

[21] C. Fung et al., "A generalized theory of an electrolyte-insulatorsemiconductor field-effect transistor," IEEE Trans. Electron Devices, vol. 33, pp. 8-18, 1986

[22] T. Prodromakis et al., "A Low-Cost Disposable Chemical Sensing Platform Based on Discrete Components," IEEE Electron Device Lett., vol. 32, no. 3, pp. 417-419, 2011.

[23] B. Palan et al., "Fundamental noise limits of ISFET-based microsystems," Proc. Eurosensors, vol. 13, pp. 169-172, 1999.

[24] T. Prodromakis et al., "Biocompatible encapsulation of CMOS based chemical sensors," Proc. IEEE Sensors, pp. 791-794, 2010.

[25] A. Ortiz-Conde, et al., "A review of recent MOSFET threshold voltage extraction methods," Elsevier Microelectronics Reliability, vol. 42, no. 4-5, pp. 583-596, 2002.

[26] B. Sheu et al., "BSIM: Berkeley short-channel IGFET model for MOS transistors," IEEE J. Solid-State Circuits, vol. 22, no. 4, pp. 558-566, 2002.

[27] E. McAdams et al., "The linear and non-linear electrical properties of the electrode-electrolyte interface," Elsevier Biosensors and Bioelectronics, vol. 10, no. 1-2, pp. 67-74, 1995.

[28] J. Davis et al., "Surface ionization and complexation at the oxide/water interface:: I. Computation of electrical double layer properties in simple electrolytes," Elsevier J. Colloid and Interface Science, vol. 63, no. 3, pp. 480-499, 1978.

[29] J. Brews and E. Nicollian, MOS physics and technology.

[30] B. Degnan et al., "Trapped charge characterization and removal on floating-gate transistors," Proc. IEEE MWSCAS, pp. 617-620, 2008.

[31] L. Bousse and P. Bergveld, "The role of buried $\mathrm{OH}$ sites in the response mechanism of inorganic-gate pH-sensitive ISFETs," Elsevier Sensors and Actuators, vol. 6, no. 1, pp. 65-78, 1984.

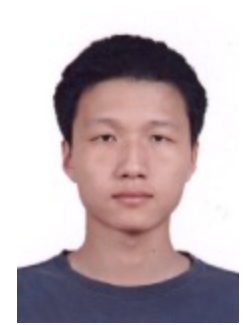

Yan Liu (AM'08) received the B.Eng degree in 2006 from Process Equipment and Control Engineering at Zhejiang University, China, and the M.Sc degree in 2007 from Electrical and Electronic Engineering at Imperial College London. He is now working towards the PhD degree in Imperial College London, focusing on the CMOS ISFETs based chemical sensing systems.

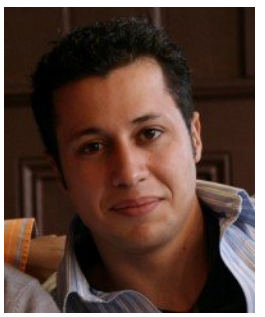

Pantelis Georgiou (AM'05-M'08) received the M.Eng. degree in Electrical and Electronic Engineering in 2004 and the Ph.D. degree in 2008 both from Imperial College London. He then moved to the Institute of Biomedical Engineering (also at Imperial) where he was appointed as a Research Fellow until joining academic faculty in 2011. He is currently a lecturer within the Department of Electrical \& Electronic Engineering and is also the head of the Bio-inspired Metabolic Technology Laboratory in the Centre for Bio-Inspired Technology and part of the Medical Engineering Solutions in Osteoarthritis Centre of Excellence. His research includes bio-inspired circuits and systems, CMOS based lab-on-chip technologies and application of micro-electronic technology to create novel medical devices. He conducted pioneering work on the silicon beta cell and is now leading the project forward to the development of the first bio-inspired artificial pancreas for Type I diabetes. Dr Georgiou is a member of the IEEE and IET. He has been elected a member of the BioCAS Technical Committee of the IEEE Circuits and Systems Society.

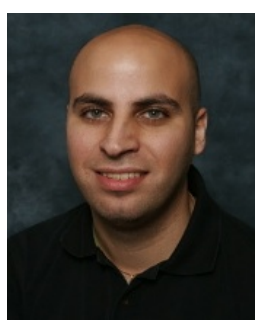

Timothy G. Constandinou (AM'98-M'01-SM'10) received the B.Eng. degree in Electrical and Electronic Engineering in 2001 and the Ph.D. degree in 2005 both from Imperial College London. He then moved to the Institute of Biomedical Engineering (also at Imperial) where he was appointed to Research Officer in Bionics until joining academic faculty in 2010 . He is currently a lecturer within the Department of Electrical \& Electronic Engineering at Imperial College London and is also the deputy director of the Centre for Bio-Inspired Technology. His Research is in the novel application of microtechnology to develop advanced medical devices (implantable, wearable and lab-on-chip) and biologicallyinspired circuits, devices and systems. Dr Constandinou is an IEEE Senior Member, an IET Member and a registered Chartered Engineer. He has been elected a member of the Sensory Systems and BioCAS Technical Committees of the IEEE Circuits \& Systems Society, and also serves on the IET awards committee. 


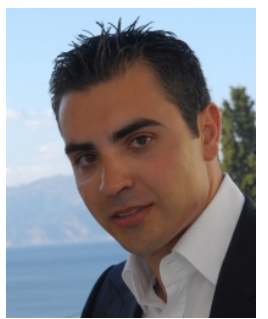

Themistoklis Prodromakis (AM'04-M'08) holds a Corrigan research fellowship in nanoscale science and technology, funded by LSI Logic Inc. and the Corrigan-Walla Foundation, within the Centre for Bio-inspired Technology at Imperial College London. He received his PhD from the Department of Electrical and Electronic Engineering at Imperial College in 2008, during which he successfully pioneered the use of interfacial polarisations for demonstrating miniature passive devices. During his research career he has contributed in several projects in the areas of RF and Microwave Design and particularly Electron Devices, including: miniaturisation techniques MEMS-based phase-shifting topologies, slow-wave filters on laminar architectures, high-k dielectrics and processing techniques for engineering polarisation mechanisms. In 2006 he contributed in setting up the Cleanroom facilities and the Microelectronics Laboratory at the IBE. He recently applied his expertise in the biomedical arena with some examples involving: the development of integrated CMOS chemical sensors, encapsulation techniques and materials and biologically inspired systems.

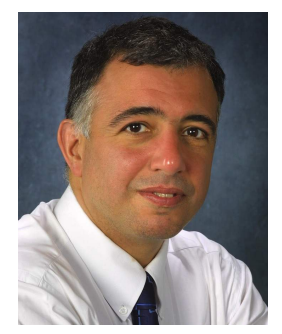

Christofer Toumazou (M'87-SM'99-F'01) is a Professor of Circuit Design, Founder and Executive Director of the Institute of Biomedical Engineering at Imperial College London, UK. Professor Toumazou has made outstanding contributions to the fields of low power analogue circuit design and current mode circuits and systems for biomedical and wireless applications. Through his extensive record of research, he has invented innovative electronic devices ranging from dual mode cellular phones to ultra-low power devices for both medical diagnosis and therapy. He has published over 320 research papers in the field of RF and low power electronics and is a member of many professional committees.. He holds 23 patents in the field, many of which are now fully granted PCT. He is the founder of four technology based companies with applications spanning ultra low-power mobile technology and wireless vital sign monitors (Toumaz Technology Ltd, UK), biomedical devices (Applied Bionics PTE, Singapore), digital audio broadcasting (FutureWaves Pte Taiwan) and DNA detection (DNA Electronics Ltd, UK). These companies employ over $50 \mathrm{RF} /$ low power engineers worldwide many of whom are Professor Toumazou's ex-graduate students. Professor Toumazou was invited to deliver the 2003 Royal Society Clifford Patterson Prize Lecture, entitled "The Bionic Man", for which he was awarded The Royal Society Clifford Patterson bronze medal. He was recently awarded the IEEE CAS Society Education Award for pioneering contributions to telecommunications and biomedical circuits and systems, and the Silver Medal from the Royal Academy of Engineering for his outstanding personal contributions to British engineering. In 2008, he was elected to the grade of Fellow of both the Royal Society and Royal Academy of Engineering. 